\title{
Electronic Population Transfer via Impulsive Stimulated X-Ray Raman Scattering with Attosecond Soft-X-Ray Pulses
}

Jordan T. O’Neal, ${ }^{1,2, *}$ Elio G. Champenois, ${ }^{1}$ Solène Oberli, ${ }^{3}$ Razib Obaid, ${ }^{4}$ Andre Al-Haddad, ${ }^{5,6}$ Jonathan Barnard, ${ }^{7}$ Nora Berrah, ${ }^{4}$ Ryan Coffee, ${ }^{1,8}$ Joseph Duris, ${ }^{9}$ Gediminas Galinis, ${ }^{7}$ Douglas Garratt, ${ }^{7}$ James M. Glownia, ${ }^{8}$ Daniel Haxton, ${ }^{10}$ Phay Ho, ${ }^{5}$ Siqi Li, ${ }^{1,2,9}$ Xiang Li,${ }^{8,12}$ James MacArthur, ${ }^{2,9}$ Jon P Marangos, ${ }^{7}$ Adi Natan, ${ }^{1}$ Niranjan Shivaram,, 13 Daniel S. Slaughter, ${ }^{13}$ Peter Walter, ${ }^{8}$ Scott Wandel, ${ }^{8}$ Linda Young, ${ }^{5,14}$ Christoph Bostedt, $, 6,15$ Philip H. Bucksbaum, ${ }^{1,2,11}$ Antonio Picón, ${ }^{3}$ Agostino Marinelli, ${ }^{1,9}$ and James P. Cryan ${ }^{1,8, \dagger}$

${ }^{1}$ Stanford PULSE Institute, SLAC National Accelerator Laboratory, Menlo Park, California 94025, USA

${ }^{2}$ Department of Physics, Stanford University, Stanford, California 94305, USA

${ }^{3}$ Departamento de Química, Universidad Autónoma de Madrid, 28049 Madrid, Spain

${ }^{4}$ Department of Physics, University of Connecticut, Storrs, Connecticut 06269, USA

${ }^{5}$ Chemical Sciences and Engineering Division, Argonne National Laboratory, Argonne, Illinois 60439, USA

${ }^{6}$ Paul-Scherrer Institute, CH-5232 Villigen PSI, Switzerland

${ }^{7}$ Blackett Laboratory, Imperial College, London SW7 2AZ, United Kingdom

${ }^{8}$ Linac Coherent Light Source, SLAC National Accelerator Laboratory, Menlo Park, California 94025, USA

${ }^{9}$ SLAC National Accelerator Laboratory, Menlo Park, California 94025, USA

${ }^{10}$ KLA Corporation, Milpitas, California 95035, USA

${ }^{11}$ Department of Applied Physics, Stanford University, Stanford, California 94305, USA

${ }^{12}$ J.R. Macdonald Laboratory, Department of Physics, Kansas State University, Manhattan, Kansas 66506, USA

${ }^{13}$ Chemical Sciences Division, Lawrence Berkeley National Laboratory, Berkeley, California 94720, USA

${ }^{14}$ Department of Physics and James Franck Institute, The University of Chicago, Chicago, Illinois 60637, USA

${ }^{15}$ LUXS Laboratory for Ultrafast X-ray Sciences, Institute of Chemical Sciences and Engineering, École Polytechnique Fédérale de Lausanne (EPFL), CH-1015 Lausanne, Switzerland

(Received 10 February 2020; revised 21 May 2020; accepted 9 July 2020; published 11 August 2020)

Free-electron lasers provide a source of $\mathrm{x}$-ray pulses short enough and intense enough to drive nonlinearities in molecular systems. Impulsive interactions driven by these $\mathrm{x}$-ray pulses provide a way to create and probe valence electron motions with high temporal and spatial resolution. Observing these electronic motions is crucial to understand the role of electronic coherence in chemical processes. A simple nonlinear technique for probing electronic motion, impulsive stimulated x-ray Raman scattering (ISXRS), involves a single impulsive interaction to produce a coherent superposition of electronic states. We demonstrate electronic population transfer via ISXRS using broad bandwidth $(5.5 \mathrm{eV}$ full width at half maximum) attosecond $\mathrm{x}$-ray pulses produced by the Linac Coherent Light Source. The impulsive excitation is resonantly enhanced by the oxygen $1 s \rightarrow 2 \pi^{*}$ resonance of nitric oxide (NO), and excited state neutral molecules are probed with a time-delayed UV laser pulse.

DOI: 10.1103/PhysRevLett.125.073203

The motion of electrons in quantum systems is fundamental to processes in chemical physics, biology, and material science. Recent experiments with attosecond temporal resolution have demonstrated that ultrafast charge motion, due to the formation of a coherent superposition of electronic states, can be tracked in simple molecular systems [1,2]. A fundamental understanding of this coherent electron motion (or charge migration) will provide crucial insight into a wide range of charge transfer and correlated electronic phenomena [3-6]. Detailed measurements are required for understanding charge migration in complex systems relevant to material science, chemistry, and biology. Nonlinear x-ray spectroscopies, which provide the capability to track the coherent electronic motion with atomic spatial localization and attosecond temporal resolution, are becoming attractive options for such measurements.

Ultrafast optical techniques are used routinely to follow chemical reactions on the femtosecond timescale. In the infrared or visible domain, nonlinear spectroscopies have led to sophisticated probes of excited state dynamics, far beyond the minimal information content of the linear response [7]. Applying these methods with sequences of $\mathrm{x}$-ray pulses will allow us to initiate and probe coherent electronic dynamics on attosecond timescales [8-12]. Furthermore, x-ray photons interact strongly with corelevel electrons; therefore, $\mathrm{x}$-ray observables are sensitive probes of the local electronic environment [13], which tracks charge motion throughout the molecule. 
The advent of x-ray free-electron lasers (XFELs) demonstrated nonlinear x-ray interactions, starting with sequential ionization of atoms and molecules [14-17]. Subsequently, direct (or nonsequential) two-photon absorption was also observed [18,19], along with nonlinear x-ray Compton scattering [20]. Among the proposed nonlinear spectroscopic techniques, stimulated x-ray Raman scattering (SXRS) is a promising method for generating nonstationary electronic states, given current XFEL capabilities [21,22].

SXRS is an inelastic $\mathrm{x}$-ray scattering process where, starting from the ground state $(g s)$ of the molecule, the $\mathrm{x}$-ray pulse first excites a core electron into an unoccupied orbital (pump transition). Before this core-excited (ce) state decays, a second interaction with the $\mathrm{x}$-ray pulse stimulates the emission of a photon driving an electron from a different valence orbital into the core vacancy (Stokes transition). This process results in the transfer of electronic population from the ground state to valence-excited (ve) neutral states. The SXRS process is shown schematically in Fig. 1(a) for the case of nitric oxide (NO). The ground state molecular orbital configuration for the nitric oxide target is $\left(1 s_{O}\right)^{2}\left(1 s_{N}\right)^{2}(3 \sigma)^{2}\left(4 \sigma^{*}\right)^{2}(5 \sigma)^{2}(1 \pi)^{4}\left(2 \pi^{*}\right)^{1}$. The nearly resonant pump transition promotes an electron from the

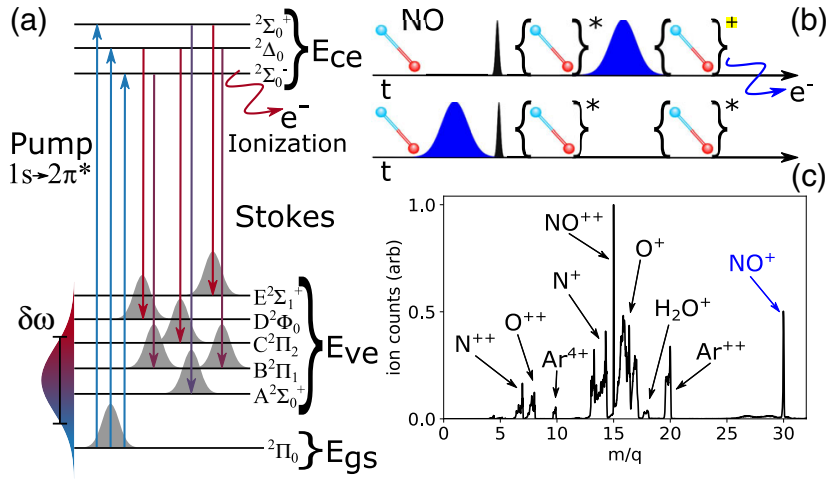

FIG. 1. (a) Schematic description of the SXRS process showing accessible states in the NO target. The pump transition is dominated by the promotion of an electron from the $1 s_{O}$ orbital to the partially occupied $2 \pi^{*}$ orbital. This configuration leads to three molecular core-excited (ce) states $\left({ }^{2} \Sigma^{+},{ }^{2} \Delta\right.$, and $\left.{ }^{2} \Sigma^{-}\right)$. The Stokes transition couples these core-excited states to a low-lying valence-excited (ve) electronic configuration, where an electron from the $1 \pi$ orbital fills the $1 s_{O}$ vacancy. We find that the dominant SXRS pathways result in populating the $B^{2} \Pi, C^{2} \Pi$, and $D^{2} \Phi$ valence-excited electronic states. The top line in panel (b) shows the pulse sequence used to probe excited state neutral molecules: An attosecond x-ray pulse (black) produces excited state NO molecules (denoted with an *) through the SXRS process. These molecules are then probed by ionization by a timedelayed (20 ns) UV pulse (blue). The bottom line shows the pulse ordering used as a reference for removing other sources of $\mathrm{NO}^{+}$ ions: The UV pulse proceeds the X-ray pulse by $5 \mathrm{~ns}$. (c) Measured ion mass-to-charge ratio $(\mathrm{m} / \mathrm{q})$ spectrum produced by the combined $\mathrm{X}$-ray and UV pulses.
$1 s_{O}$ orbital to the partially occupied $2 \pi^{*}$ orbital, leading to three molecular core-excited states: ${ }^{2} \Sigma^{+},{ }^{2} \Delta$, and ${ }^{2} \Sigma^{-}$. The Stokes transition could populate many valence-excited electronic configurations, but we find that the dominant pathway involves an electron from the $1 \pi$ orbital filling the $1 s_{O}$ vacancy. The dominant SXRS pathways populate the $B^{2} \Pi, C^{2} \Pi$, and $D^{2} \Phi$ electronic states, each with the $(1 \pi)^{3}\left(2 \pi^{*}\right)^{2}$ excited state configuration.

The total population transfer $(S)$ following the inelastic scattering process is described by a Kramers-Heisenberg expression (in atomic units),

$$
S\left(\omega_{0}\right)=\sum_{\mathrm{ve}}\left|T_{\mathrm{ve}, \mathrm{gs}}^{(2)}\left(\omega_{0}\right)\right|^{2} \delta\left(\Delta \omega-\omega_{\mathrm{ve}, \mathrm{gs}}\right)
$$

where $\omega_{0}$ is the central photon energy of the x-ray pulse, $\Delta \omega=\omega-\omega^{\prime}$ is the difference between the pump and Stokes frequency, and $T^{(2)}$ is the second-order transition matrix element. The length gauge expression for the second-order transition matrix element (or transition polarizability [10]) for a single broad bandwidth x-ray pulse is given by

$$
\begin{aligned}
T_{\mathrm{ve}, \mathrm{gs}}^{(2)}\left(\omega_{0}\right)= & \frac{1}{2 \pi} \sum_{\mathrm{ce}}\left\langle\psi_{\mathrm{ve}}|\vec{\epsilon} \cdot \vec{r}| \psi_{\mathrm{ce}}\right\rangle\left\langle\psi_{\mathrm{ce}}|\vec{\epsilon} \cdot \vec{r}| \psi_{\mathrm{gs}}\right\rangle \\
& \times \int d \omega \int d \omega^{\prime} \frac{E^{*}(\omega) E\left(\omega^{\prime}\right)}{\omega+\omega_{0}-\omega_{\mathrm{ce}, \mathrm{gs}}+i \Gamma_{\mathrm{ce}, \mathrm{gs}}},
\end{aligned}
$$

where $\mathcal{E}(t)=\operatorname{Re}\left[\int d \omega E(\omega) e^{-i \omega_{0} t}\right]$ is the time-dependent electric field and $\vec{\epsilon}$ is the polarization vector. The coreexcited states decay via Auger electron emission with a lifetime of $\left(\Gamma_{\mathrm{ce}, \mathrm{gs}}\right)^{-1}$ in atomic units. The $\delta$ function in Eq. (1) enforces energy conservation between the emitted and absorbed photons $(\Delta \omega)$, and the initial and final states of the molecule $\left(\omega_{\mathrm{ve}, \mathrm{gs}}\right)$. The denominator in Eq. (2) leads to atomic site-specific enhancements when the central energy of the x-ray pulse is tuned close to a near-edge resonance $\left(\omega_{\mathrm{ce}, \mathrm{gs}}\right)$.

An early experiment by Weninger et al. demonstrated SXRS through resonant excitation of a dense neon target with femtosecond XFEL pulses [21]. The X-ray pulses, having a partially coherent bandwidth of $7 \mathrm{eV}$, stimulated the Raman process, and the emitted light was amplified in the core-excited gas column. Following the successful demonstration in an atomic target, subsequent experiments focused on molecular targets [22]. Using the Raman amplification technique, the Raman gain for a molecular system is expected to be much smaller than in the atomic case [23]. Despite substantial experimental effort, no Raman gain has been observed in molecular systems using femtosecond x-ray pulses [22,23].

In this Letter, we extend SXRS to the impulsive limit using attosecond soft $\mathrm{x}$-ray pulses. In the impulsive limit, the exciting pulse is short compared to the natural time evolution of the system, and thus the excitation is initially 
localized near a specific atomic site and will evolve on attosecond timescales [24]. In impulsive SXRS (ISXRS), both the pump and Stokes frequencies are within the coherent bandwidth of the incident $\mathrm{x}$-ray pulse, and any valence-excited state within the $\mathrm{x}$-ray pulse bandwidth can be populated. Moreover, a second x-ray pulse could be used to probe the time evolution of the electronic wave packet using a two-color scheme, which was already demonstrated by Duris et al. [25].

The enhanced SASE (ESASE) method [26], implemented at the Linac Coherent Light Source (LCLS), generates soft $\mathrm{x}$-ray pulses near the oxygen $K$-edge $(540 \mathrm{eV})$ with large coherent bandwidth $(\sim 5.5 \mathrm{eV})$ and corresponding pulse duration of approximately 500 attoseconds [25]. These isolated attosecond pulses have energies in the range of 10-300 $\mu \mathrm{J}$ and, when focused, can produce intensities above $\sim 10^{18} \mathrm{~W} / \mathrm{cm}^{2}$, sufficient for driving nonlinear $\mathrm{x}$-ray interactions. The previous attempts to observe SXRS in molecular systems focused on amplification of the Stokes emission line; however, in this study we detect the transfer of electronic population to valenceexcited states via photoemission spectroscopy. The demonstration of ISXRS in a gas-phase molecule is a stepping stone toward studying electronic dynamics in complex systems, such as large biological molecules and condensed phase systems.

Extending this work to the condensed phase will be experimentally challenging. High intensity $\mathrm{x}$-ray pulses cause significant sample damage. In addition, the photoemission detection scheme used in our demonstration will be much harder to implement for condensed phase targets. To some extent, sample damage could be avoided by raster scanning a solid target or using high-velocity liquid sheets to replenish the target between laser pulses. Reducing the $\mathrm{x}$-ray intensity while increasing the pulse repetition rate would also help to mitigate damage problems. The reduction in single-shot signal levels would be offset by increased data rates, which will be useful for gas phase experiments as well. Despite these difficulties, the information provided by ISXRS would lead to important insights into attosecond dynamics in large systems.

We carried out measurements at the atomic, molecular, and optical science (AMO) experimental hutch at the LCLS using the LAMP end station [27]. A double-sided velocity map imaging (VMI) spectrometer simultaneously captured electrons and ions produced via photoionization of a skimmed molecular beam of NO. A large background of electrons produced by stray light reduced the sensitivity of the electron VMI below the expected signal levels; thus, in the present experiments, only the ion VMI spectrometer could be used, and it was optimized to produce high resolution ion time-of-flight spectra. Broad bandwidth $\mathrm{x}$-ray pulses were tuned to the vicinity of the oxygen $1 s \rightarrow$ $2 \pi^{*}$ resonance of $\mathrm{NO}$ at $532.6 \mathrm{eV}$ [28]. The $2 \pi^{*}$ resonance consists of three overlapping states, with characters shown in Fig. 1(a). The x-ray pulses transfer population between the ground and various valence-excited states using this core-excited resonance as an intermediate. A number of low-lying excited states of NO are accessible within the spectral bandwidth of the attosecond x-ray pulses. Moreover, three of these states are long-lived: They have fluorescence lifetimes in excess of $100 \mathrm{~ns}$ [29], they exhibit a sharp vibrational progression in the visible absorption spectrum [30], and our molecular dynamics simulations show that a significant fraction of the excited state population does not dissociate (see Supplemental Material [31]).

The measurement scheme, depicted in Fig. 1(b), probes the number of valence-excited molecules produced by interaction with an attosecond $\mathrm{x}$-ray pulse by time-delayed ( $20 \mathrm{~ns})$ ionization with a weak, ultraviolet (UV) probe pulse. The intensity of the UV laser pulse is adjusted close to the two-photon ionization threshold of ground state molecules. At these intensities, ionization of ground state molecules is nearly negligible, but valence-excited states are ionized efficiently, yielding singly charged ions. Therefore, the yield of this ion channel is related to the valence-excited state fraction. To quantify other sources of parent ions, background measurements are performed where the UV pulse precedes the x-ray pulse by $5 \mathrm{~ns}$. The background measurements are interleaved as three out of seven consecutive shots. The difference in ion yield between the two pulse configurations is proportional to the valence-excited state fraction produced by the x-ray pulse.

$\mathrm{NO}^{+}$ions are discriminated based on their charge-tomass ratio, which is derived from the time-of-flight in our ion spectrometer. The time of arrival of photoions is measured by analyzing the raw waveform from the microchannel plate (MCP) detector in the ion spectrometer. The resulting ion spectrum is shown in Fig. 1(c). In this experiment, the event rate for ions in the $\mathrm{NO}^{+}$ $(m / q=30)$ channel is about 0.22 ions per shot. We use the additional spatial information provided by the position sensitive anode detector [27] to count the number of ions produced when multiple ions overlap in the time-of-flight signal, as discussed in the Supplemental Material [31].

We scan the $x$-ray central photon energy in the range $531-551 \mathrm{eV}$ by adjusting the electron beam energy. This range includes the $1 s \rightarrow 2 \pi^{*}$ resonance as well as the oxygen $K$-edge. Figure 2(a) shows a Gaussian smoothed histogram of the total $\mathrm{NO}^{+}$ion yield as a function of central photon energy (dashed red), which shows the energetic position of the $1 s \rightarrow 2 \pi^{*}$ resonance. Here we have normalized the total yield to the pulse energy per shot and the number of shots at each photon energy.

We estimate the average bandwidth of the attosecond $\mathrm{x}$-ray pulses by convolving the known shape of the resonant absorption feature [28] with a Gaussian of increasing spectral width until the convolved curve matches the measured $\mathrm{NO}^{+}$yield curve in Fig. 2. We retrieve an 


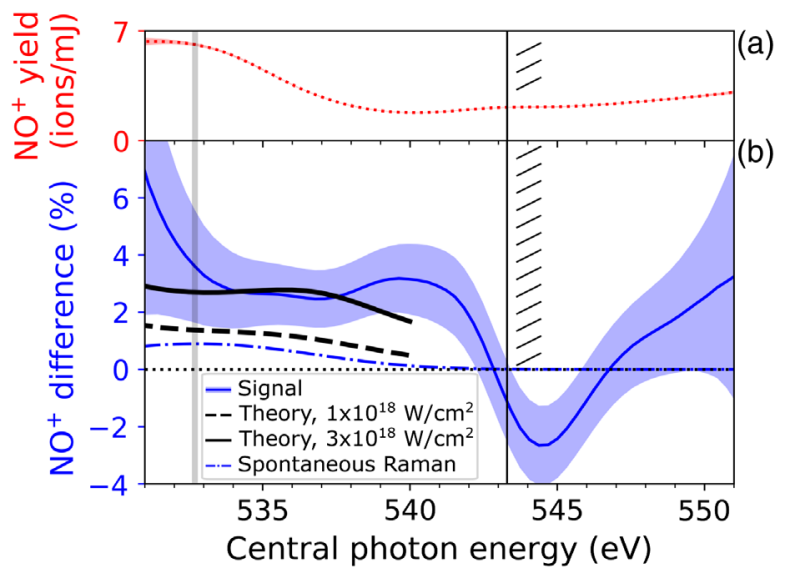

FIG. 2. (a) Central photon energy dependence of the total yield of $\mathrm{NO}^{+}$ions. The total $\mathrm{NO}^{+}$yield demonstrates the energetic position of the oxygen $1 s \rightarrow 2 \pi^{*}$ resonant feature (vertical gray bar). (b) Central photon energy dependence of $\mathrm{NO}^{+}$difference signal (solid blue), Gaussian smoothed for clarity $(1.5 \mathrm{eV}$ at FWHM) with a $1 \sigma$ error bar (blue shaded area). This difference signal is directly proportional to the number of valence-excited state neutral molecules produced by the x-ray pulse. A vertical dashed bar shows the energy position of the oxygen $K$-edge. We compare the measured yield with results from our simulations for peak intensities of $10^{18} \mathrm{~W} / \mathrm{cm}^{2}$ (dashed black) and $3 \times 10^{18} \mathrm{~W} / \mathrm{cm}^{2}$ (solid black), along with the expected spontaneous Raman rate (dot-dashed blue).

average bandwidth of $5.5 \pm 0.3 \mathrm{eV}$. This average bandwidth is consistent with single-shot $\mathrm{x}$-ray spectra measured in a separate set of experiments [25].

To analyze the fraction of valence-excited states created by the x-ray pulse, we consider the differential ion yield,

$$
\Delta Y_{\mathrm{NO}^{+}}=2 \times \frac{Y_{+}-Y_{-}}{Y_{+}+Y_{-}},
$$

taken between shots where the UV laser pulse arrives after the X-ray pulse $\left(Y_{+}\right)$and the UV laser pulse arrives before the $\mathrm{x}$-ray pulse $\left(Y_{-}\right)$. Figure 2(b) (solid blue curve) plots the differential ion yield, $\Delta Y_{\mathrm{NO}^{+}}$, as a function of x-ray central photon energy. Neutral excited states appear in the region of the $2 \pi^{*}$ resonance, measured as a $2.6 \pm 0.6 \%$ increase in the $\mathrm{NO}^{+}$yield, or $4.1 \sigma$ above the null result. This excess is significantly above the predicted rate of spontaneous X-ray Raman scattering calculated in the Supplemental Material [31], shown as a dot-dashed blue curve in Fig. 2. The broad range of photon energies over which these neutral excited states are observed, extending from below the $2 \pi^{*}$ resonance out to the oxygen $K$-edge, is characteristic of an impulsive excitation. When the interaction period is extremely short, the importance of the energy detuning in the denominator of Eq. (2) becomes less important, which would lead to a broad spectral region where it is possible to drive ISXRS, limited mostly by the $\mathrm{x}$-ray bandwidth. We also notice in Fig. 2 that the measured yield of excited state neutral molecules drops sharply just before the oxygen $K$-edge, where the bandwidth of the $\mathrm{X}$-ray pulse can ionize the oxygen $1 s$ electron and linear $\mathrm{x}$-ray photoionization competes with the ISXRS process. Above the oxygen $K$-edge, the curve shows variability similar to the background channels, as discussed in the Supplemental Material [31].

We model the ISXRS process using the effective threestate model described in Ref. [37]. Numerical simulations of the ISXRS process are performed by solving the timedependent Schrödinger equation for a fixed nuclear geometry using the energy of the ground, core-excited, and valence-excited states computed to second order in multiconfiguration perturbation theory (CASPT2) with the OpenMolcas program [38]. The calculation is performed for fixed-in-space molecules, here the x-ray polarization is oriented perpendicular to the molecular axis. We assume the $\mathrm{x}$-ray pulses have a Fourier transform-limited pulse duration and a corresponding spectral bandwidth of $5.5 \mathrm{eV}$. Our simulation models direct single-photon ionization as a population loss mechanism, in addition to the Auger decay of core-excited states [37]. The primary loss mechanism is the Auger decay of core-excited states that persist after the exciting x-ray pulse is over; however, the losses due to single-photon ionization of the valence shell and nitrogen $K$-shell become important for intensities above $10^{18} \mathrm{~W} / \mathrm{cm}^{2}$, which is shown in the Supplemental Material [31].

The time-dependent electronic populations calculated for a pulse with a central photon energy of $532.6 \mathrm{eV}$ are shown in Fig. 3(a). The population transfer into the valenceexcited states is due exclusively to ISXRS. Following the initial excitation, the core-excited states decay due to the Auger process, whereas the valence-excited states are much more stable. However, on longer timescales ( $>10$ 's fs), many of these states dissociate. The states with significant bound populations are the $\Phi_{0}, \Pi_{1}$, and $\Pi_{2}$ states. To determine the dissociation fraction for each state, we extend the simulation to $1 \mathrm{ps}$ at each intensity (results are shown in the Supplemental Material) and find the bound fraction to be roughly $30 \%, 90 \%$, and $4 \%$ for the $\Phi_{0}, \Pi_{1}$, and $\Pi_{2}$ states, respectively. This survival probability has only a minor dependence on the pulse intensity.

Figure 3(b) shows the population fraction transferred into each valence-excited state $3 \mathrm{fs}$ after the x-ray pulse arrives. The maximal population transfer occurs at a photon energy that is slightly red-detuned from the $2 \pi^{*}$ resonance because the Stokes transition is several $\mathrm{eV}$ below the resonance position. This maximum depends on the intensity and shifts closer to the original resonance for larger intensities due to ac Stark shifting of the transition frequencies. Our simulations show that ISXRS coherently populates multiple valence-excited states and thus provides a method for generating a coherent electronic wave packet. While the results shown in Fig. 3 assume a fixed nuclear 

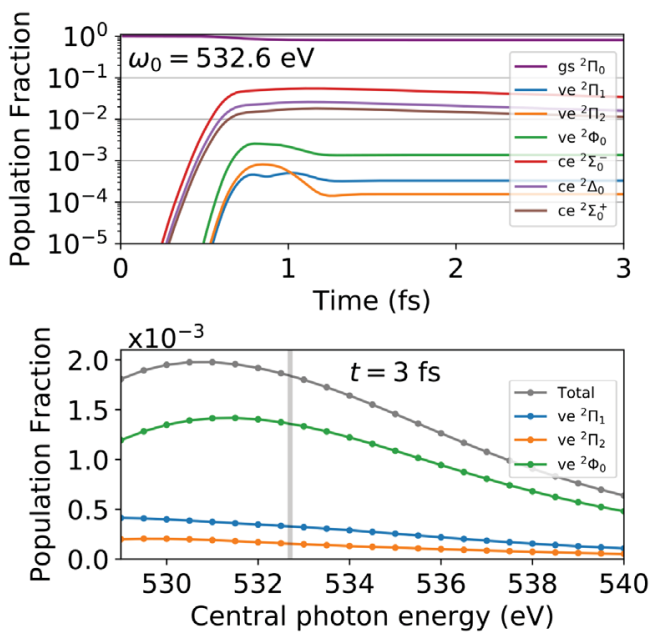

FIG. 3. (a) Time evolution of electronic populations during and after interaction with an attosecond x-ray pulse. The x-ray pulse has a central photon energy of $532.6 \mathrm{eV}$, on resonance with the ${ }^{2} \Pi_{0} \rightarrow{ }^{2} \Delta_{0}$ transition, and a peak intensity of $3 \times 10^{18} \mathrm{~W} / \mathrm{cm}^{2}$. The results are intensity averaged over the entire focal volume. The possible final states are labeled by their character, in addition to being specified as ground (gs), valence-excited (ve), or coreexcited states (ce). (b) Valence-excited state populations $3 \mathrm{fs}$ after excitation as a function of central photon energy of the incident $\mathrm{X}$-ray pulse. A peak intensity of $3 \times 10^{18} \mathrm{~W} / \mathrm{cm}^{2}$ is used. The vertical gray bar indicates the energy position simulated in panel (a).

geometry, simulations incorporating nuclear dynamics in the core-excited state did not significantly alter the results (see the Supplemental Material [31] for an example including nuclear dynamics).

The experimental observable [percent change in differential ion yield, Eq. (3)] measures the ratio between the valence-excited state population and the number of $\mathrm{NO}^{+}$ ions produced by the pump and probe pulses. To accurately compare the simulated excited state fractions from Fig. 3 with the experimentally observed quantity, we must also model all sources of $\mathrm{NO}^{+}$ions. $\mathrm{NO}^{+}$ions originate from direct interaction with the x-ray pulse via Auger decay of core-excited molecules and direct valence ionization, which can be estimated directly from the time-dependent electronic state population calculations. Additionally, the $\mathrm{UV}$ laser pulse can produce $\mathrm{NO}^{+}$via two-photon ionization, which is measured directly in the experiment. The UV laser also contributes to the $\mathrm{NO}^{+}$ion yield via singlephoton ionization of valence-excited, neutral molecules produced either through ISXRS or spontaneous Raman scattering. The ISXRS contribution is taken from the total excited state population in Fig. 3(b) after accounting for molecular dissociation, whereas the rate for spontaneous Raman scattering is estimated in the Supplemental Material [31] and is shown in Fig. 2(b) as a dot-dashed blue line. Computing the expected differential ion with Eq. (3), we obtain the black curves in Fig. 2.
Assuming a $2-\mu \mathrm{m}$ diameter $\mathrm{x}$-ray focal spot $\left(\sim 3 \mu \mathrm{m}^{2}\right)$, an $\mathrm{x}$-ray pulse duration of roughly 500 as, and an x-ray mirror reflectance between $10 \%$ and $50 \%$, we expect an x-ray peak intensity in the range $10^{17}-10^{18} \mathrm{~W} / \mathrm{cm}^{2}$. From comparing the measured and calculated differential ion yield (Fig. 2), we estimate the x-ray peak intensity of about $3 \times 10^{18} \mathrm{~W} / \mathrm{cm}^{2}$. At this intensity, both the total signal strength and central photon energy dependence near the $2 \pi^{*}$ resonance show good agreement with the measurement. The strong dependence on intensity could be leveraged to precisely measure the $\mathrm{x}$-ray intensity in future experiments. As the central photon energy approaches the oxygen $K$-edge, core-excited Rydberg states can contribute to the ISXRS yield. These intermediate states are not included in the simulation, and thus the predicted $\mathrm{NO}^{+}$yield decreases as the bandwidth begins to reach above $540 \mathrm{eV}$.

Beyond intensity calibration, the ISXRS measurement is made relative to the $\mathrm{NO}^{+}$yield, and thus we can compute a two-photon cross section by using the $\mathrm{x}$-ray intensity found by matching the data to simulations. By convolving the $\mathrm{x}$-ray bandwidth with the absolute cross section for singlephoton absorption from Ref. [39], we can conclude that $\sigma^{(2)}=(3 \pm 2) \times 10^{-55} \mathrm{~cm}^{4} \mathrm{~s} /$ photon, for a Gaussian pulse with 5.5-eV spectral bandwidth. This is within an order of magnitude of the two-photon absorption cross sections previously measured in the $\mathrm{x}$-ray regime after accounting for the $Z^{-4}$ scaling $[14,18,19]$.

In this Letter, we have observed an excess of valenceexcited molecules following interaction with broad bandwidth, attosecond, and soft x-ray pulses tuned near a preedge resonance. We compared the photon energy dependence of the excitation fraction with simulations of spontaneous Raman and ISXRS and found the data to be consistent with the expected level of the ISXRS signal. From these measurements, we have estimated the ISXRS cross section. Moreover, our theoretical calculations show that a superposition of valence-excited states is created. This observation of nonlinear $\mathrm{x}$-ray physics with isolated attosecond pulses opens a path for investigations of transient electronic phenomena using $\mathrm{x}$-ray free-electron lasers. Future experiments should employ two-color attosecond X-ray pulses [25] to follow the electronic coherence initiated by impulsive Raman pumping, which will allow electronic motion to be disentangled from nuclear motion, going beyond recent experiments employing femtosecond pulses [40]. This methodology would enable site-specific probing of valence electron motion with high temporal resolution through nonlinear x-ray interactions. Another future direction is toward extending single-pulse coherent Raman scattering spectroscopies to the $\mathrm{x}$-ray regime as a site-specific probe of photochemical reactions [41]. Measurements of this type will provide insight into the role of electronic coherence in photochemical reactions.

We thank Matthew Ware for insightful discussions during the data analysis. Use of the Linac Coherent 
Light Source (LCLS), SLAC National Accelerator Laboratory, is supported by the U.S. Department of Energy (DOE), Office of Science, Office of Basic Energy Sciences (BES) under Contract No. DE-AC0276SF00515. This work was primarily supported by the AMOS program in the Chemical Sciences, Geosciences, and Biosciences Division of BES at the DOE. The AMOS program also supported A. A. H., P. H., C. B., L. Y. through Contract No. DE-AC02-06CH11357, N.B. and R. O. through Grant No. DE-SC0012376, and N.S. and D. S. S. through Contract No. DE-AC02-05-CH11231. This work was also supported by the DOE-BES Accelerator and detector research program. S. O., A. P. acknowledge funding support from grant ref. RTI2018-097355-A-I00 (MCIU/AEI/FEDER, UE), CM grant ref. 2017-T1/IND5432, and computing resources from CCC-UAM. J. B., G. G., D. G., J. P. M. gratefully acknowledge funding support from UK EPSRC grants No. EP/R019509/1 and No. EP/ I032517/1.

* Corresponding author. joneal2@stanford.edu ${ }^{\dagger}$ Corresponding author. jcryan@slac.stanford.edu

[1] F. Calegari, D. Ayuso, A. Trabattoni, L. Belshaw, S. D. Camillis, S. Anumula, F. Frassetto, L. Poletto, A. Palacios, P. Decleva, J. B. Greenwood, F. Martín, and M. Nisoli, Ultrafast electron dynamics in phenylalanine initiated by attosecond pulses, Science 346, 336 (2014).

[2] P. M. Kraus, B. Mignolet, D. Baykusheva, A. Rupenyan, L. Horný, E. F. Penka, G. Grassi, O. I. Tolstikhin, J. Schneider, F. Jensen, L. B. Madsen, A. D. Bandrauk, F. Remacle, and H. J. Wörner, Measurement and laser control of attosecond charge migration in ionized iodoacetylene, Science 350, 790 (2015).

[3] S. R. Leone, C. W. McCurdy, J. Burgdörfer, L. S. Cederbaum, Z. Chang, N. Dudovich, J. Feist, C. H. Greene, M. Ivanov, R. Kienberger, U. Keller, M. F. Kling, Z.-H. Loh, T. Pfeifer, A. N. Pfeiffer, R. Santra, K. Schafer, A. Stolow, U. Thumm, and M. J. J. Vrakking, What will it take to observe processes in 'real time'?, Nat. Photonics 8, 162 (2014).

[4] F. Lépine, M. Y. Ivanov, and M. J. J. Vrakking, Attosecond molecular dynamics: Fact or fiction?, Nat. Photonics 8, 195 (2014).

[5] G. D. Scholes, G. R. Fleming, L. X. Chen, A. AspuruGuzik, A. Buchleitner, D. F. Coker, G. S. Engel, R. van Grondelle, A. Ishizaki, D. M. Jonas, J. S. Lundeen, J. K. McCusker, S. Mukamel, J. P. Ogilvie, A. Olaya-Castro, M. A. Ratner, F. C. Spano, K. B. Whaley, and X. Zhu, Using coherence to enhance function in chemical and biophysical systems, Nature (London) 543, 647 (2017).

[6] H. J. Wörner, C. A. Arrell, N. Banerji, A. Cannizzo, M. Chergui, A. K. Das, P. Hamm, U. Keller, P. M. Kraus, E. Liberatore, P. Lopez-Tarifa, M. Lucchini, M. Meuwly, C. Milne, J.-E. Moser, U. Rothlisberger, G. Smolentsev, J. Teuscher, J. A. van Bokhoven, and O. Wenger, Charge migration and charge transfer in molecular systems, Struct. Dyn. 4, 061508 (2017).

[7] S. S. Mukamel, Principles of Nonlinear Optical Spectroscopy (Oxford University Press, Oxford, 1995).

[8] J. D. Biggs, Y. Zhang, D. Healion, and S. Mukamel, Two-dimensional stimulated resonance Raman spectroscopy of molecules with broadband x-ray pulses, J. Chem. Phys. 136, 174117 (2012).

[9] J. D. Biggs, Y. Zhang, D. Healion, and S. Mukamel, Watching energy transfer in metalloporphyrin heterodimers using stimulated X-ray Raman spectroscopy, Proc. Natl. Acad. Sci. U.S.A. 110, 15597 (2013).

[10] S. Mukamel, D. Healion, Y. Zhang, and J. D. Biggs, Multidimensional attosecond resonant $\mathrm{X}$-ray spectroscopy of molecules: Lessons from the optical regime, Annu. Rev. Phys. Chem. 64, 101 (2013).

[11] S. Tanaka and S. Mukamel, Coherent X-Ray Raman Spectroscopy: A Nonlinear Local Probe for Electronic Excitations, Phys. Rev. Lett. 89, 043001 (2002).

[12] S. Tanaka and S. Mukamel, Probing exciton dynamics using Raman resonances in femtosecond x-ray four-wave mixing, Phys. Rev. A 67, 033818 (2003).

[13] K. Siegbahn, Electron spectroscopy for atoms, molecules, and condensed matter, Rev. Mod. Phys. 54, 709 (1982).

[14] L. Young et al., Femtosecond electronic response of atoms to ultra-intense x-rays, Nature (London) 466, 56 (2010).

[15] J. P. Cryan et al., Auger Electron Angular Distribution of Double Core-Hole States in the Molecular Reference Frame, Phys. Rev. Lett. 105, 083004 (2010).

[16] L. Fang, M. Hoener, O. Gessner, F. Tarantelli, S. T. Pratt, O. Kornilov, C. Buth, M. Gühr, E. P. Kanter, C. Bostedt, J. D. Bozek, P. H. Bucksbaum, M. Chen, R. Coffee, J. Cryan, M. Glownia, E. Kukk, S. R. Leone, and N. Berrah, Double Core-Hole Production in $\mathrm{N}_{2}$ : Beating the Auger Clock, Phys. Rev. Lett. 105, 083005 (2010).

[17] M. Hoener et al., Ultraintense X-Ray Induced Ionization, Dissociation, and Frustrated Absorption in Molecular Nitrogen, Phys. Rev. Lett. 104, 253002 (2010).

[18] G. Doumy et al., Nonlinear Atomic Response to Intense Ultrashort X Rays, Phys. Rev. Lett. 106, 083002 (2011).

[19] S. Ghimire, M. Fuchs, J. Hastings, S. C. Herrmann, Y. Inubushi, J. Pines, S. Shwartz, M. Yabashi, and D. A. Reis, Nonsequential two-photon absorption from the K shell in solid zirconium, Phys. Rev. A 94, 043418 (2016).

[20] M. Fuchs et al., Anomalous nonlinear x-ray Compton scattering, Nat. Phys. 11, 964 (2015).

[21] C. Weninger, M. Purvis, D. Ryan, R. A. London, J. D. Bozek, C. Bostedt, A. Graf, G. Brown, J. J. Rocca, and N. Rohringer, Stimulated Electronic X-Ray Raman Scattering, Phys. Rev. Lett. 111, 233902 (2013).

[22] V. Kimberg, A. Sanchez-Gonzalez, L. Mercadier, C. Weninger, A. Lutman, D. Ratner, R. Coffee, M. Bucher, M. Mucke, M. Agåker, C. Såthe, C. Bostedt, J. Nordgren, J. E. Rubensson, and N. Rohringer, Stimulated X-ray Raman scattering a critical assessment of the building block of nonlinear x-ray spectroscopy, Faraday Discuss. 194, 305 (2016).

[23] N. Rohringer, X-ray Raman scattering: A building block for nonlinear spectroscopy, Phil. Trans. R. Soc. A 377, 20170471 (2019). 
[24] I. V. Schweigert and S. Mukamel, Probing valence electronic wave-packet dynamics by all x-ray stimulated Raman spectroscopy: A simulation study, Phys. Rev. A 76, 012504 (2007).

[25] J. Duris et al., Tunable isolated attosecond x-ray pulses with gigawatt peak power from a free-electron laser, Nat. Photonics 14, 30 (2020).

[26] A. A. Zholents, Method of an enhanced self-amplified spontaneous emission for x-ray free electron lasers, Phys. Rev. ST Accel. Beams 8, 040701 (2005).

[27] T. Osipov, C. Bostedt, J.-C. Castagna, K. R. Ferguson, M. Bucher, S. C. Montero, M. L. Swiggers, R. Obaid, D. Rolles, A. Rudenko, J. D. Bozek, and N. Berrah, The LAMP instrument at the Linac coherent light source freeelectron laser, Rev. Sci. Instrum. 89, 035112 (2018).

[28] N. Kosugi, J. Adachi, E. Shigemasa, and A. Yagishita, Highresolution and symmetry-resolved $\mathrm{N}$ and OK-edge absorption spectra of NO, J. Chem. Phys. 97, 8842 (1992).

[29] T. Hikida, T. Suzuki, and Y. Mori, Fluorescence lifetime studies of $\mathrm{NO} \mathrm{A}{ }^{2} \Sigma^{+}(\nu=5, N=9), \mathrm{B}^{2} \Pi_{3 / 2} \quad(\nu=8$, $J=8.5), \quad \mathrm{C}^{2} \Pi_{3 / 2} \quad(\nu=1, \quad J=8.5)$, and $\mathrm{D}^{2} \Sigma^{+} \quad(\nu=0$, $N=5$ ), Chem. Phys. 118, 437 (1987).

[30] W. F. Chang, G. Cooper, and C. E. Brion, Absolute optical oscillator strengths for the photoabsorption of nitric oxide (5-30 eV) at high resolution, Chem. Phys. 170, 111 (1993).

[31] See Supplemental Material at http://link.aps.org/ supplemental/10.1103/PhysRevLett.125.073203 for a more detailed description of the data and theory analysis, which includes Refs. [32-36].

[32] J. H. Hubbell, P. N. Trehan, N. Singh, B. Chand, D. Mehta, M. L. Garg, R. R. Garg, S. Singh, and S. Puri, A review, bibliography, and tabulation of $\mathrm{K}, L$, and higher atomic shell X-ray fluorescence yields, J. Phys. Chem. Ref. Data 23, 339 (1994).

[33] T. X. Carroll, M. Coville, P. Morin, and T. D. Thomas, Deexcitation electron spectroscopy of core-excited NO as a function of excitation energy, J. Chem. Phys. 101, 998 (1994).

[34] P. Erman, A. Karawajczyk, E. Rachlew-Källne, and C. Strömholm, Photoionization and photodissociation of nitric oxide in the range 9-35 eV, J. Chem. Phys. 102, 3064 (1995).

[35] H. J. Werner, P. J. Knowles, G. Knizia, F. R. Manby, and M. Schütz, Molpro: A general-purpose quantum chemistry program package, Comput. Mol. Sci. 2, 242 (2012).

[36] G. Remmers, M. Domke, A. Puschmann, T. Mandel, G. Kaindl, E. Hudson, and D. A. Shirley, High-resolution inner-shell photoionization of NO, Chem. Phys. Lett. 214, 241 (1993).

[37] A. Picón, J. Mompart, and S. H. Southworth, Stimulated Raman adiabatic passage with two-color x-ray pulses, New J. Phys. 17, 083038 (2015).

[38] I. Fdez. Galván et al., OpenMolcas: From source code to insight, J. Chem. Theory Comput. 15, 5925 (2019).

[39] J. Berkowitz, Atomic and Molecular Photoabsorption: Absolute Total Cross Sections (Academic Press, New York, 2002), p. 350.

[40] A. Picón et al., Hetero-site-specific x-ray pump-probe spectroscopy for femtosecond intramolecular dynamics, Nat. Commun. 7, 11652 (2016).

[41] H. Frostig, O. Katz, A. Natan, and Y. Silberberg, Singlepulse stimulated Raman scattering spectroscopy, Opt. Lett. 36, 1248 (2011). 